\title{
Predicting Atrial Fibrillation Recurrence After Catheter Ablation Through Time Variability of P-wave Features
}

\author{
Antonio Ruiz ${ }^{1}$, Miguel A Arias ${ }^{2}$, Alberto Puchol $^{2}$, Marta I Pachón ${ }^{2}$, José J Rieta ${ }^{3}$, Raúl Alcaraz ${ }^{1}$ \\ ${ }^{1}$ Research Group in Electronic, Biomedical and Telecommunications Engineering, \\ University of Castilla-La Mancha, Cuenca, Spain \\ ${ }^{2}$ Cardiac Arrhythmia Department, Hospital Virgen de la Salud, Toledo, Spain \\ ${ }^{3}$ BioMIT.org, Electronic Engineering Department, Universitat Politecnica de Valencia, Spain
}

\begin{abstract}
Nowadays, the first-line therapy for paroxysmal atrial fibrillation (PAF) is pulmonary vein isolation through catheter ablation. However, the success rate of this procedure is still not as high as desirable. Thus, preoperative prediction of early $A F$ recurrence after ablation is a challenge to select optimal candidates for the intervention. To this end, some promising predictors based on the P-wave in short ECG signals have been proposed in the last years. However, evolution of the P-wave along the time has still not been analyzed. Hence, the present work studies how time variability of two features of the $P$-wave predicts midterm cryoablation failure. For 45 PAF patients, a standard 12-lead ECG signal was obtained for 5 minutes before ablation. An automatic algorithm was then used to delineate all P-waves in lead II, and duration and amplitude were computed. The resulting time series were characterized by their mean, standard deviation and coefficient of variation $(\mathrm{CV})$. Correlating these measures with ablation outcome, the $C V$ for both parameters obtained the best discrimination between patients. In fact, compared with the mean value, the CV for both features obtained accuracies 10\% greater, thus achieving values of $70 \%$. These outcomes entail that time variability of the $P$-wave can reveal new information about the proarrhythmic condition of the patients, thus improving predictions of ablation failure.
\end{abstract}

\section{Introduction}

Atrial fibrillation (AF) is a common arrhythmia in elderly population, which is related to other major cardiovascular diseases, such as stroke and heart failure[1]. The mechanisms initiating and maintaining this arrhythmia are not completely understood yet [2], but it is today accepted that a common source triggering $\mathrm{AF}$ is the existence of heterogenous electrical activity in muscle sleeves surrounding the pulmonary veins (PV) [3]. Hence, their isolation via catheter ablation is nowadays the cornerstone for the treatment of symptomatic paroxysmal AF [3]. For that purpose, a common procedure is radiofrequency ablation, which consists of applying current around PV in a pointby-point fashion to provoke permanent lesions by heating [4]. Nonetheless, cryoablation has recently received extensive attention, since it can be applied in a less complex and faster way, and moreover is able to obtain similar mid- and long-term success rates [5]. In this case, a balloon is used to apply cryogenic energy around each PV in a single-step, thus causing atrial lesions by freezing [4].

Although both types of procedures have reported initial rates of effectiveness about $80-90 \%$ [6], AF recurrence is common after some weeks or months. In fact, two or three procedures are required in about $50 \%$ of patients [6]. This result occurs even patients are treated with potent antiarrhythmic drugs after ablation, and complete and stable PV isolation is reached [5]. Consequently, beyond PV reconnection after ablation, other causes can trigger AF recurrence, including the existence of an advanced atrial substrate remodeling as well as of foci in atrial areas different from PV [7]. Within this scenario, preoperative anticipation of AF recurrence after ablation plays a key role in selecting optimal candidates for the procedure and thus make the management of AF patients more efficient [5].

For that purpose, some echocardiographic predictors, such atrial velocity conduction or left atrial diameter, have been proposed [5]. However, they have only reported a limited predictive ability, far from being acceptable from a clinical point of view. More recently, other authors have tried to evaluate the proarrhythmic condition of the patients by analyzing P-wave reflected on the surface ECG recording [5]. They hypothesized that extensive atrial remodeling provokes elongated atria, as well as conduction delays, thus leading to longer and smaller P-waves [5]. Accordingly, some $\mathrm{P}$-wave features, including duration, morphology, dispersion, and peak time, among others, have reported predictive abilities of mid- and long-term abla- 
tion outcome about 60-70\% [5]. Moreover, a few works have also corroborated that these $\mathrm{P}$-wave parameters are significantly modified after ablation [8].

However, moderate degrees of atrial remodeling could only alter negligibly P-wave morphology and therefore the analysis of how this waveform evolves along the time has been suggested for a better assessment of the proarrhythmic condition after catheter ablation [9]. Thus, the present work aims at analyzing whether time variability of two common P-wave features, i.e., amplitude and duration, can reach a better estimation of atrial remodeling, and consequently a better anticipation of mid-term cryoablation failure, in patients with paroxysmal AF.

\section{Database}

A total of 45 patients (14 women and 31 men) suffering from paroxysmal AF and underwent cryoballon catheter ablation were enrolled in the study. Shortly, the ablation protocol started by sedating the patient with conscious sedation or general anesthesia. Next, catheters were introduced by femoral venous access and transeptal puncture was used to access to the left atrium. Anticoagulation with heparin was maintained and dosed during all the intervention using proper monitoring. Finally, to isolate each PV, one or several (if required) applications of cryothermal energy were performed. When isolation of all PVs was successfully completed, the procedure finished and the patient was continuously monitored for some hours. If no complications occurred for this time, the patient was discharged.

Initially, full isolation of PVs was achieved in all patients and sinus rhythm (SR) properly restored. Nonetheless, although the patients did not presented any complication for 9 months of follow-up and were under antiarrhythmic and anticoagulant treatment, 14 of them relapsed to $\mathrm{AF}$.

\section{Methods}

\subsection{Data preprocessing}

For 5 minute before ablation, a standard 12-lead ECG signal was acquired from every patient with a sampling rate of $1 \mathrm{kHz}$ and 16 bits of resolution. Because the $\mathrm{P}$-wave presents a larger amplitude and a more stable monophasic morphology in lead II compared with the remaining ones [10], it was only analyzed. Thus, the signal was initially preprocessed to eliminate powerline interference, high-frequency noise, baseline wander. More precisely, a previously published wavelet-based denoising method was used for removal of the powerline interference [11]. Although some high-frequency noise was also removed with this algorithm, a low-pass filtering with cut-off frequency of $70 \mathrm{~Hz}$ was also used to obtain a completely clean sig- nal [12]. Finally, the baseline wander was extracted by low-pass filtering the signal with a cut-off frequency of $0.8 \mathrm{~Hz}$ and then subtracted [12].

\subsection{Time variability of the $P$-wave}

Fiducial points of every P-wave, i.e., its onset, peak and offset, were identified using a recently published methodology [13]. It is based on generating a gaussian model of every P-wave for making its delineation easier, as well as on the use of an adaptive slope threshold for taking into consideration the morphology of the preceding P-waves. After exhaustive validation with different public and manually annotated databases, the method have provided a sensitivity about $94 \%$ in detecting P-waves, as well as a limited location error lower than $12 \mathrm{~ms}$ between the annotations obtained automatically and those provided by experts [14]. These results overcome those obtained by other previously proposed $\mathrm{P}$-wave delineators [14], thus providing reliable information. Nonetheless, delineation of all $\mathrm{P}$ waves was visually supervised, and clear errors were manually corrected.

Finally, duration and amplitude of all P-waves were obtained from their fiducial points, and the resulting times series were characterized by computing values of mean, standard deviation (std), and coefficient of variation (CV). This last measure was obtained as the ratio of std to mean.

\subsection{Performance assessment}

For all parameters proposed to predict mid-term cryoablation failure, results are expressed as mean \pm std. Additionally, statistical differences between the patients who relapsed to $\mathrm{AF}$ and maintained SR during the follow-up were estimated through a Mann-Whitney U test. A twotailed value of $p<0.05$ was considered as statistically significant.

On the other hand, the ability of each predictor to discern between both groups of patients was assessed through a receiver operating characteristic (ROC) curve. This plot is the result of plotting the fraction of true positives out of positives (sensitivity) against the fraction of false positives out of the negatives ( 1 -specificity) at various threshold settings. Sensitivity (Se) was considered as the percentage of patients who relapsed to AF correctly classified, while specificity $(\mathrm{Sp})$ was the rate of patients maintaining SR properly identified. The optimal threshold was selected as the one that provided the highest percentage of patients rightly detected, i.e. accuracy (Acc).

\section{4. $\quad$ Results}

Table 1 shows mean and std values obtained for the three measures computed from the time series of P-wave dura- 
Table 1. Values of mean, std and CV obtained from the time series of P-wave duration and amplitude

\begin{tabular}{llccc}
\hline \hline & & \multicolumn{4}{c}{ Group of patients } \\
\cline { 3 - 5 } P-wave & Metric & maintaining SR & relapsing to AF & $p$-value \\
\hline \multirow{3}{*}{ Durat. } & Mean & $154.7 \pm 21.4 \mathrm{~ms}$ & $156.3 \pm 30.7 \mathrm{~ms}$ & 0.607 \\
& Std & $15.1 \pm 5.3 \mathrm{~ms}$ & $21.4 \pm 9.6 \mathrm{~ms}$ & 0.020 \\
& $\mathrm{CV}$ & $9.7 \pm 3.0 \%$ & $13.4 \pm 4.7 \%$ & 0.003 \\
\hline \multirow{4}{*}{ Amplit. } & Sean & $71.7 \pm 28.3 \mu \mathrm{V}$ & $57.9 \pm 19.6 \mu \mathrm{V}$ & 0.211 \\
& $\mathrm{CV}$ & $11.3 \pm 0.5 \mu \mathrm{V}$ & $12.4 \pm 0.6 \mu \mathrm{V}$ & 0.447 \\
\hline \hline
\end{tabular}

Table 2. Classification results for the measures computed from the time series of $\mathrm{P}$-wave duration and amplitude.

\begin{tabular}{llccc}
\hline \hline P-wave & Parameter & Se (\%) & Sp (\%) & Acc (\%) \\
\hline \multirow{3}{*}{ Duration } & Mean & 57.1 & 61.4 & 60.0 \\
& Std & 71.4 & 71.0 & 71.1 \\
& CV & 71.4 & 74.2 & 73.3 \\
\hline \multirow{4}{*}{ Amplitude } & Mean & 64.3 & 52.6 & 56.6 \\
& Std & 57.1 & 54.8 & 55.6 \\
& CV & 64.3 & 64.5 & 64.4 \\
\hline \hline
\end{tabular}

tion and amplitude. Of note is that patients maintaining SR exhibited a mean value of the P-wave duration lower than those relapsing to AF, however no statistically significant differences were seen. In contrast, differences between groups were statistically significant both for std and $\mathrm{CV}$ values computed from the the time series of P-wave duration. In this case, patients relapsing to AF also showed larger values than those who maintained SR.

In regard to the time series of P-wave amplitude, a notably overlapping was noticed for the values of means and std obtained for both groups of patients. In fact, the only parameter noticing statistically significant differences between the patients who relapsed to AF and maintained SR during the follow-up was the CV. For this measure, notably larger values were observed for the patients relapsing to AF than for those who maintained SR.

Finally, classification results for the analyzed measures from the times series of P-wave duration and amplitude are displayed in Table 2. As can be seen for the case of the $\mathrm{P}$-wave duration, the best discriminant ability was reported by the parameters std and $\mathrm{CV}$. Both indices obtained values of Acc larger than $70 \%$, moreover exhibiting comparable values of Se and Sp. Regarding the P-wave amplitude, the best outcome was obtained by the CV. Nonetheless, values of Se, Sp and Acc lower than in the previous case, around $65 \%$, were noticed. The two other indices, i.e. mean and std, only showed values of Acc lower than $57 \%$.

\section{Discussion}

Previous works have revealed a prolonged P-wave duration as a promising predictor of long-term ablation failure [5]. In fact, values of P-wave duration larger than 120-150 ms have achieved to discriminate between patients with and without AF recurrence during the first year post-ablation with a diagnostic accuracy about 70\% [5]. Additionally, the presence of P-waves with low amplitude before ablation has also been associated with an increased likelihood of AF recurrence, a threshold of $0.1 \mathrm{mV}$ also reaching values of sensitivity and specificity around $70 \%$ [15]. In comparison with these outcomes, classification rates between 10 and $15 \%$ lower have been obtained by mean values computed for the time series of P-wave duration and amplitude. The widely different methodologies used to estimate the P-wave features could justified this conflicting results. Thus, in previous works signalaveraged ECG recordings or very short 12-lead ECG signals were used to assess $\mathrm{P}$-wave duration and amplitude $[5,15]$, whereas in the present study these parameters were obtained by averaging those values obtained beat-bybeat from a 5 minute-length interval of standard lead II.

Although no statistically significant differences between patients who maintained SR and relapsed to AF during the follow-up were noticed for mean values of P-wave duration and amplitude, a tendency toward P-waves with higher amplitude and shorter duration was noticed for the patients who maintained SR for months after ablation. This observation is consistent with previous results $[5,15]$, thus suggesting the presence of more moderate atrial remodeling before catheter ablation may enhance the likelihood of AF recurrence in mid- and long-term, even as isolation of all PVS was successfully completed.

Compared to the mean value, the parameters std and $\mathrm{CV}$ obtained from the time series of both P-wave amplitude and duration yielded notably larger and statistically significant differences between groups of patients, thus reaching improvements in classification higher than $10 \%$. This outcome points to the idea that the P-wave evolution along the time could quantify more accurately moderate levels of atrial remodeling. Thus, more invariant and rigid atrial electrical conduction found in patients able to maintain SR for long time could be reflected on a more limited time variability in the $\mathrm{P}$-wave amplitude and duration, thus suggesting a greater likelihood to successfully respond to catheter cryoablation. Nonetheless, this interesting finding should be corroborated in further studies, where wider databases will have to be analyzed.

\section{Conclusions}

This pioneer work has characterized time variability of the P-wave morphology to preoperatively predict the mid- 
term failure of catheter cryoablation. In comparison with the mean value, the standard deviation and coefficient of variation of the time series obtained for both P-wave amplitude and duration have exhibited improvements about $10 \%$ in anticipation of patients with and without AF recurrence during the first 9 months post-ablation. Consequently, the analysis of how the P-wave morphology evolves along the time may provide novel information about the proarrhythmic condition of the AF patients before ablation, which could help for a better selection of the optimal candidates for this treatment.

\section{Acknowledgments}

This research has been supported by the grants DPI2017-83952-C3 from MINECO/AEI/FEDER EU, SBPLY/17/180501/ 000411 from Junta de Comunidades de Castilla-la Mancha and AICO/2019/036 from Generalitat Valenciana

\section{References}

[1] Lippi G, Sanchis-Gomar F, Cervellin G. Global epidemiology of atrial fibrillation: An increasing epidemic and public health challenge. Int J Stroke Jan 2020; 1747493019897870.

[2] Schotten U, Dobrev D, Platonov PG, Kottkamp H, Hindricks G. Current controversies in determining the main mechanisms of atrial fibrillation. J Intern Med May 2016; 279(5):428-38.

[3] Cheniti G, Vlachos K, Pambrun T, Hooks D, Frontera A, Takigawa M, Bourier F, Kitamura T, Lam A, Martin C, Dumas-Pommier C, Puyo S, Pillois X, Duchateau J, Klotz N, Denis A, Derval N, Jais P, Cochet H, Hocini M, Haissaguerre M, Sacher F. Atrial fibrillation mechanisms and implications for catheter ablation. Front Physiol 2018;9:1458.

[4] Yano M, Egami Y, Yanagawa K, Nakamura H, Matsuhiro Y, Yasumoto K, Tsuda M, Okamoto N, Tanaka A, MatsunagaLee Y, Yamato M, Shutta R, Nishino M, Tanouchi J. Comparison of myocardial injury and inflammation after pulmonary vein isolation for paroxysmal atrial fibrillation between radiofrequency catheter ablation and cryoballoon ablation. J Cardiovasc Electrophysiol Jun 2020;31(6):13151322.

[5] Pranata R, Yonas E, Vania R. Prolonged P-wave duration in sinus rhythm pre-ablation is associated with atrial fibrillation recurrence after pulmonary vein isolation-A systematic review and meta-analysis. Ann Noninvasive Electrocardiol 09 2019;24(5):e12653.

[6] Inaba O, Metzner A, Rottner L, Mathew S, Lemes C, Maurer T, Heeger C, John A, Hashiguchi N, Wohlmuth P,
Ouyang F, Kuck KH, Rillig A, Reissmann B. Radiofrequency or cryoballoon ablation for index pulmonary vein isolation: What is the impact on long-term clinical outcomes after repeat ablation? J Cardiovasc Electrophysiol May 2020;31(5):1068-1074.

[7] Redfearn DP, Skanes AC, Gula LJ, Griffith MJ, Marshall HJ, Stafford PJ, Krahn AD, Yee R, Klein GJ. Noninvasive assessment of atrial substrate change after wide area circumferential ablation: a comparison with segmental pulmonary vein isolation. Ann Noninvasive Electrocardiol Oct 2007;12(4):329-37.

[8] Furniss GO, Panagopoulos D, Kanoun S, Davies EJ, Tomlinson DR, Haywood GA. The effect of atrial fibrillation ablation techniques on $\mathrm{P}$-wave duration and $\mathrm{P}$-wave dispersion. Heart Lung Circ Mar 2019;28(3):389-396.

[9] Huo Y, Holmqvist F, Carlson J, Gaspar T, Hindricks G, Piorkowski C, Bollmann A, Platonov PG. Variability of Pwave morphology predicts the outcome of circumferential pulmonary vein isolation in patients with recurrent atrial fibrillation. J Electrocardiol 2015;48(2):218-25.

[10] De Bacquer D, Willekens J, De Backer G. Long-term prognostic value of $\mathrm{P}$-wave characteristics for the development of atrial fibrillation in subjects aged 55 to 74 years at baseline. Am J Cardiol Sep 2007;100(5):850-4.

[11] García M, Martínez-Iniesta M, Ródenas J, Rieta JJ, Alcaraz R. A novel wavelet-based filtering strategy to remove powerline interference from electrocardiograms with atrial fibrillation. Physiol Meas 11 2018;39(11):115006.

[12] Sörnmo L, Laguna P. ECG Signal Processing in Bioelectrical Signal Processing in Cardiac and Neurological Applications. 1st edition. Elsevier Academic Press, 2005. ISBN 978-0-12-437552-9.

[13] González F, Alcaraz R, Rieta JJ. Electrocardiographic Pwave delineation based on adaptive slope gaussian detection. In Computing in Cardiology. IEEE, 2017; 1-4.

[14] Ruiz A, Arias MA, Pachón MI, Langley P, Rieta JJ, Alcaraz $\mathrm{R}$. Thorough assessment of a P-wave delineation algorithm through the use of diverse electrocardiographic databases. In 2019 E-Health and Bioengineering Conference (EHB). IEEE, 2019; 1-4.

[15] Park JK, Park J, Uhm JS, Joung B, Lee MH, Pak HN. Low $\mathrm{P}$-wave amplitude $(<0.1 \mathrm{mV})$ in lead $\mathrm{I}$ is associated with displaced inter-atrial conduction and clinical recurrence of paroxysmal atrial fibrillation after radiofrequency catheter ablation. Europace Mar 2016;18(3):384-91.

Address for correspondence:

Antonio Ruiz Moreno

E.S.I. Informática., Campus Univ., 02071, Albacete, Spain

Phone: +34-967-599-200

e-mail: antonio.ruiz@uclm.es 